\title{
Changes in acute hospital costs after employing clinical facilitators to improve stroke care in Victoria, Australia
}

\author{
Dominique A. Cadilhac ${ }^{1,2,3^{*}}$ D, Helen M. Dewey ${ }^{2,4}$, Sonia Denisenko ${ }^{3}$, Christopher F. Bladin ${ }^{2,4}$ and Atte Meretoja $2,5,6$
}

\begin{abstract}
Background: Hospital costs for stroke are increasing and variability in care quality creates inefficiencies. In 2007, the Victorian Government (Australia) employed clinical facilitators for three years in eight public hospitals to improve stroke care. Literature on the cost implications of such roles is rare. We report changes in the costs of acute stroke care following implementation of this program.

Methods: Observational controlled before-and-after cohort design. Standardised hospital costing data were compared pre-program (financial year 2006-07) and post-program (2010-11) for all admitted episodes of stroke or transient ischaemic attack (TIA) using ICD-10 discharge codes. Costs in Australian dollars (AUD) were adjusted to a common year 2010. Generalised linear regression models were used for adjusted comparisons.

Results: A 20\% increase in stroke and TIA episodes was observed: 2624 pre-program (age > 75 years: 53\%) and 3142 postprogram (age > 75 years: 51\%); largely explained by more TIA admissions (up from 785 to 1072). Average length of stay reduced by $22 \%$ (pre-program 7.3 days to post-program 5.7 days, $p<0.001$ ). Six hospitals provided cost data. Average perepisode costs decreased by 10\% (pre-program AUD7888 to post-program AUD7115). After adjusting for age, sex, stroke type, and hospital, average per-episode costs decreased by $6.1 \%$ from pre to post program $(p=0.025)$. When length of stay was additionally adjusted for, these costs increased by $10.8 \%$, indicating a greater mean cost per day $(p<0.001)$.
\end{abstract}

Conclusion: Cost containment of acute inpatient episodes was observed after the implementation of stroke clinical facilitators, likely associated with the shorter lengths of stay.

Keywords: Stroke, Cost-benefit analysis, Healthcare, Policy

\section{Background}

Stroke is a high cost condition, and the majority of stroke-related costs arise from hospitalisation. In the first year, approximately $30 \%$ of costs for stroke are attributable to acute hospital care and $30 \%$ to inpatient rehabilitation $[1,2]$. With the expected increases in the incidence of stroke associated with population ageing, finding more effective and cost-effective methods of providing hospital care for acute stroke within an environment of limited resources is important.

\footnotetext{
* Correspondence: dominique.cadilhac@monash.edu

${ }^{1}$ Translational Public Health and Evaluation Division, Stroke and Ageing Research, Department of Medicine, School of Clinical Sciences at Monash Health, Monash University, Clayton, Australia

${ }^{2}$ Stroke Division, Florey Institute of Neuroscience and Mental Health,

University of Melbourne, Heidelberg, Australia

Full list of author information is available at the end of the article
}

Currently, the most successful and universally applicable intervention for acute stroke in reducing death and disability is organised management in stroke units [3]. Stroke units support greater adherence to evidencebased interventions via a dedicated and specialised interdisciplinary clinical team making them more efficient than general wards $[4,5]$. However, in Australia just over half of all patients with stroke receive care in a stroke unit and access to other evidence-based treatments remains variable [6].

Providing a dedicated position for co-ordination or facilitation of uptake of evidence-based care in hospital settings, including the establishment of stroke units, may be one solution to improve stroke care outcomes $[7,8]$. Clinical leadership and facilitation, (i.e. where an

(c) The Author(s). 2019 Open Access This article is distributed under the terms of the Creative Commons Attribution 4.0 International License (http://creativecommons.org/licenses/by/4.0/), which permits unrestricted use, distribution, and reproduction in any medium, provided you give appropriate credit to the original author(s) and the source, provide a link to the Creative Commons license, and indicate if changes were made. The Creative Commons Public Domain Dedication waiver (http://creativecommons.org/publicdomain/zero/1.0/) applies to the data made available in this article, unless otherwise stated. 
individual with the appropriate knowledge and skills supports clinicians to change their practice), are cornerstones for enhancing the uptake of evidence [9]. The role of clinical facilitators may include establishing improved systems of communication, providing education and training to upskill staff, reviewing gaps in the provision of evidencebased practice and working with clinicians to improve the quality of care using evidence-based strategies, such as implementing clinical protocols $[10,11]$. However, evidence for the effectiveness of clinical facilitators in the field of stroke remains limited [12]. In our previous work we determined that the fixed-term employment of facilitators was effective in positively influencing stroke care in hospitals (e.g. more patients receiving brain imaging within $24 \mathrm{~h}$, greater access to care in a stroke unit and intravenous thrombolysis if an ischaemic stroke) [13]. It remains unknown if there are broader benefits to hospitals associated with facilitator programs. Our aim was to describe the costs of acute care before and after the implementation of the stroke clinical facilitator program in Victoria, Australia.

\section{Methods}

\section{Setting and context}

In Australia, the funding of acute public hospital care is the responsibility of the state government. The Victorian Stroke Clinical Network (VSCN) was established by the Victorian government in 2007. The VSCN comprised an interdisciplinary executive committee of clinicians and government representatives, who meet monthly, to provide board direction for policy and strategies to improve stroke care including advancing recommendations within the Stroke Care Strategy for Victoria. Priorities included improving access to stroke units, thrombolysis, and use of evidence-based care protocols [14]. The VSCN executive endorsed the employment of clinical facilitators for a time-limited period (i.e. 2 to 3 years) in selected priority hospitals based on the results of a national audit, number of patients admitted per year or if in a regional location. The national audit program of acute services is undertaken every two years and is used to monitor adherence to Australian stroke clinical guidelines. At each participating hospital the medical records of up to 40 consecutive patients admitted with a primary diagnosis of stroke are retrospectively audited for patient demographic information, adherence to recommended processes of care and hospital-based patient outcomes [15]. These data were used to identify priority hospitals requiring support to improve adherence to clinical processes of care [13].

From May 2008, these 'Stroke Network Facilitators' (herein referred to as Facilitators) commenced in their roles to support the establishment of better systems of care for stroke in the priority hospitals, including establishing stroke units and clinical protocols [12]. These
Facilitators had clinical backgrounds in nursing or allied health disciplines and all were employed half time (e.g. 2.5 days per week), with the exception being one large regional hospital where two Facilitators provided fulltime cover [12]. The hospitals included three metropolitan sites within Melbourne (Hospital $[\mathrm{H}]$ identification: $\mathrm{H} 4, \mathrm{H} 6, \mathrm{H} 8$ ) and five regional sites in other locations within Victoria (Fig. 1). Only three of these hospitals (H1, H2, H5) had a formalised acute stroke unit prior to the Facilitators commencing in their role [12]. The annual cost of employing Facilitators ranged from $\$ 45,000$ to $\$ 60,000$ between 2008 and 2011 per Facilitator if working 2.5 days per week (overall $\sim \$ 530,000$ per year). The cost of employing facilitators was borne by the Victorian Department of Health and not the hospitals. Therefore, these costs were not taken into account as part of the analysis, which was based on anonymised patient-level clinical costing data from hospitals.

\section{Study design}

This was an observational controlled before-and-after cohort design study from the perspective of hospitals. Standardised hospital episode and clinical costing data, which were anonymised and approved for use for a secondary purpose, were supplied by the Victorian Government, and were compared pre-program (financial year [FY] 2006-07) and post-program (FY 2010-11). Eligibility for inclusion in these analyses was based on hospital discharge International Classification of Diseases (ICD) 10 codes for stroke and transient ischaemic attack (TIA). Ischaemic stroke was defined as I63, intracerebral haemorrhage (ICH) as I61, TIA as G45 (excluding G45.4 Transient global amnesia), and Stroke, not specified (for any particular type) as I64. All data received were based on episodes of care and de-identified at an individual patient-level. Therefore, a single person may have had multiple episodes of care for stroke in the dataset. Based on data from the Australian Stroke Clinical Registry this is approximately $4 \%$ [16]. Further, we were unable to link episodes and produce estimates for individual patients because a unique personal identifier does not exist in Australia. Only Victorian Admitted Episodes Data linked to clinical costing data were used.

Most, but not all hospitals provide clinical costing data as part of the annual Victorian cost-weights study [17]. All salaries and wages are allocated to different categories including nursing, medical, allied health, emergency care, theatre, pharmacy, pathology, imaging, intensive care or coronary care and other. Medical supplies, pharmaceuticals, pathology equipment, imaging and hotel expenses are also allocated [18]. Indirect costs, including administration, research and training are also attributed to the most appropriate cost category (please see Additional file 1). Six of the eight participating hospitals 


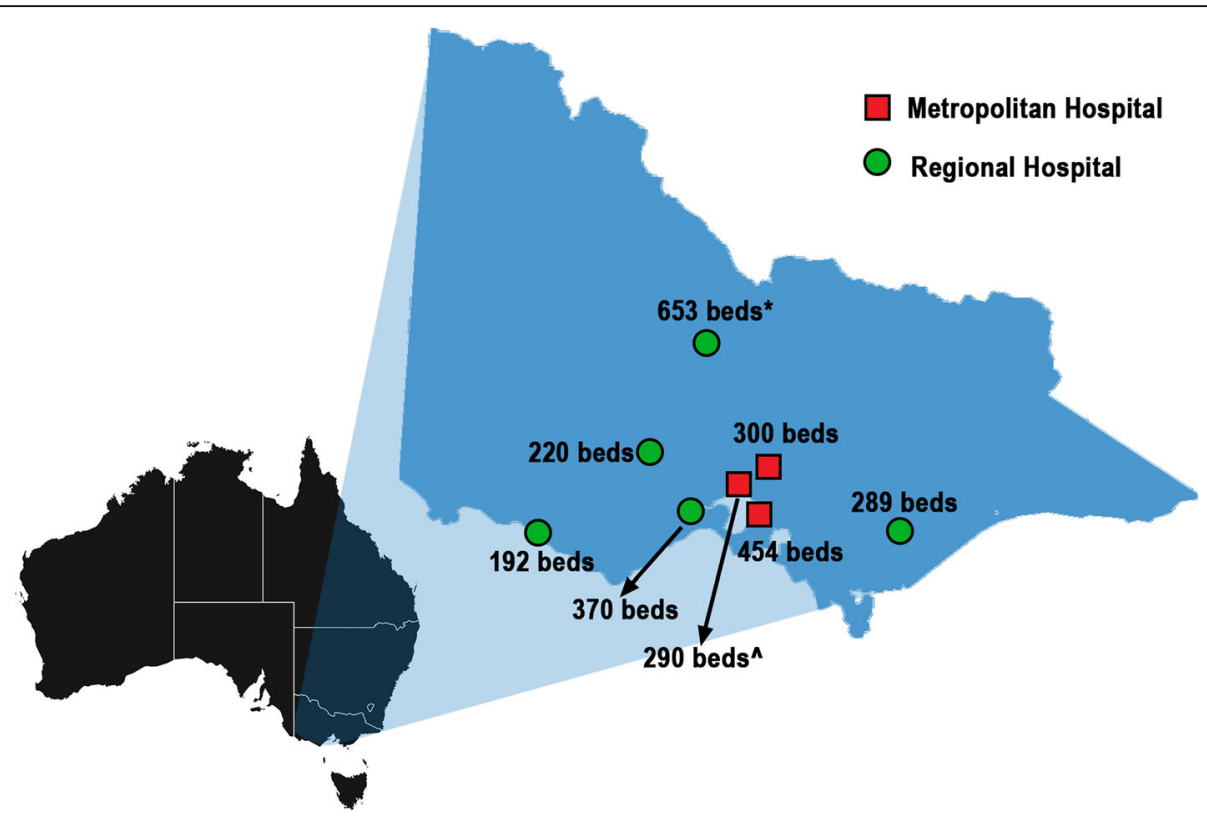

Fig. 1 Location of participating hospitals. The map depicted in Fig. 1 was created by the authors by adapting images freely available online. The map of Australia was sourced from: https://pixabay.com/en/australia-continent-geography-map-23497/ and the map of Victoria was sourced from: https://pixabay.com/en/victoria-map-australia-state-23535/ Hospital bed numbers as accessed 10th January 2017 https://www2.health.vic.gov.au/ hospitals-and-health-services/public-hospitals-victoria $\wedge$ Recent relocation of some services to another campus within health service; ${ }^{*}$ includes on site in-patient rehabilitation beds

provided costing data for the present study through the Victorian Government. This was because there were delays in submission of clinical costing data to the Victorian Government, from hospitals $\mathrm{H} 7$ and $\mathrm{H} 8$, and therefore data from one metropolitan and one regional hospital were unavailable within the time frame of this project. The costs of employing Facilitators were not included.

\section{Data analysis}

The data were analysed using SPSS Statistics Software version 22 (IBM Corp., Armonk, NY). Descriptive statistics, using parametric and non-parametric methods appropriate for the data, were used, as well as generalised linear regression modelling of costs with gamma distributions and log linked for multivariable analyses that adjusted for age, sex, stroke type, and length of stay. Costs were adjusted (i.e. inflated) to a common reference year using the total health price index of 1.0813 for inflating $2006-07$ prices to 2010 11 prices [19]. To convert costs to United States dollars please multiply by 1.5 which was the purchasing power parity in 2011 [20].

\section{Results}

The number of episodes of care in 2010-11 increased in all hospitals, on average by $20 \%$ since 2006-07 (Table 1). The median number of episodes contributed by the hospitals in 2006-07 was 248 (interquartile range [IQR]:
157-522) and for 2010-11 was 283 (IQR: 208-594). The three hospitals located in the Melbourne metropolitan local government area had a greater number of episodes in both periods with the exception being $\mathrm{H} 2$ which is located in the most populous regional area outside of Melbourne (Table 1). Age distributions for the comparison periods are presented in Fig. 2. Overall, there was a $43 \%$ increase in the number of patients with stroke or TIA admitted who were aged under 55 years, $21 \%$ increase if aged 55-64 years, $17 \%$ increase among those aged 65 to 74 years, 9\% increase if aged $74-84$ years, and $27 \%$ increase among those aged $85+$ years. Age and sex distributions were also similar between periods for the different stroke types (Additional file 1: Table S1).

Table 2 provides information on the number of episodes, length of stay, total costs and the average cost per episode in each comparison period by stroke type and overall. In 2010-11 there were greater numbers of TIA episodes and fewer strokes classified as type 'not specified'. Despite the increasing numbers of episodes, the length of stay was significantly reduced over time, by an average of $22 \%$ (Table 2). A one day reduction in median length of stay between 2006 and 07 (median 4; IQR 1-9 days) and 2010-11 (median 3; IQR 1-7 days) $(p<0.001)$ was observed. This is mainly explained by shorter lengths of stay for ischaemic stroke (2006-07 median 7; IQR 4-13 days vs. 2010-11 median 5; IQR 3-9 days; $\mathrm{p}<0.001)$ and no change over time was 
Table 1 Total episodes of care by acute hospital, stroke type and year

\begin{tabular}{|c|c|c|c|c|c|c|c|c|c|c|c|}
\hline \multirow{2}{*}{$\begin{array}{l}\text { Hospital ID } \\
\text { Financial Year }\end{array}$} & \multicolumn{2}{|c|}{ Ischaemic stroke } & \multicolumn{2}{|c|}{ Intracerebral haemorrhage } & \multicolumn{2}{|c|}{ Stroke, not specified } & \multicolumn{2}{|l|}{ TIA } & \multicolumn{3}{|l|}{ Total } \\
\hline & $2006-07$ & $2010-11$ & $2006-07$ & 2010-11 & $2006-07$ & $2010-11$ & $2006-07$ & 2010-11 & 2006-07 & 2010-11 & $\Delta \%$ \\
\hline \multicolumn{12}{|c|}{ Hospitals in regional Victorian locations } \\
\hline $\mathrm{H} 1$ & 65 & 101 & 21 & 25 & 29 & 27 & 47 & 60 & 162 & 213 & $31 \%$ \\
\hline $\mathrm{H} 2$ & 242 & 269 & 59 & 66 & 42 & 50 & 154 & 184 & 497 & 569 & $14 \%$ \\
\hline $\mathrm{H} 3$ & 44 & 73 & 17 & 21 & 52 & 45 & 29 & 52 & 142 & 191 & $35 \%$ \\
\hline $\mathrm{H} 7$ & 70 & 79 & 15 & 20 & 54 & 51 & 56 & 103 & 195 & 253 & $30 \%$ \\
\hline $\mathrm{H} 5$ & 44 & 45 & 15 & 17 & 26 & 29 & 37 & 55 & 122 & 146 & $20 \%$ \\
\hline \multicolumn{12}{|c|}{ Hospitals in Melbourne metropolitan location } \\
\hline H6 & 242 & 407 & 49 & 79 & 134 & 77 & 171 & 226 & 596 & 789 & $32 \%$ \\
\hline $\mathrm{H} 4$ & 153 & 142 & 25 & 31 & 30 & 35 & 92 & 104 & 300 & 312 & $4 \%$ \\
\hline $\mathrm{H} 8$ & 250 & 249 & 72 & 53 & 89 & 79 & 199 & 288 & 610 & 669 & $10 \%$ \\
\hline Total & 1110 & 1365 & 273 & 312 & 456 & 393 & 785 & 1072 & 2624 & 3142 & $20 \%$ \\
\hline Total $\Delta \%$ & & $23 \%$ & & $14 \%$ & & $-14 \%$ & & $37 \%$ & & $20 \%$ & \\
\hline
\end{tabular}

$H$ hospital, TIA transient ischaemic attack, $\Delta \%$ percentage change from 2006 to 07 to $2010-2011$, ID hospital identification number

noted in median values for length of stay among patients admitted with ICH (2006-07 median 3; IQR $1-9$ days vs. 2010-11 median 3; IQR $1-7$ days; $p=0.136$ ).

The analysed episodes reflected a total of 19,037 patient bed-days (total episodes/total number of days) in these acute hospitals in 2006-2007 with a 7\% decrease to 17,763 bed-days in 2010-2011. The proportion of beds occupied at any given time by patients with $\mathrm{ICH}$ remained stable at 11.2 to $11.4 \%$ while the proportion of ischaemic stroke was reduced from 61.9 to $60.8 \%$, and the proportion of patients with TIA increased from 11.6 to $15.5 \%$.

Additional results at an individual hospital level and aggregated by stroke type, where relevant, are provided in (Additional file 1: Tables S1-S4).

\section{Differences in hospital costs}

Overall, the six hospitals with clinical costing data spent AUD13.5 million in the care of these patients in 2006-7 and AUD15.6 million in 2010-11, a 16\% increase (Table 2). However, the average per-episode costs decreased by $10 \%$ over the 4years (2006-7 AUD7888 versus 2010-11 AUD7115) (Table 2). After adjusting for patient age, sex, stroke type, and hospital, there was a $6.1 \%$ decrease in average per-episode costs between 2006 and 2010 (95\% confidence interval 0.9 to $11.4 \% ; p=0.022$ ). However, if length of stay was additionally adjusted for, a $10.8 \%$ increase in total cost was observed (95\% confidence interval 7.2 to $14.3 \%, p<0.001)$, illustrating the increase in cost per day. In other words, the average cost per episode decreased by $6.1 \%$, but the average cost per day increased by $10.8 \%$.

Hospitals with the largest reductions in length of stay (Additional file 1: Table S2) had evidence of the most marked cost containment as shown in Additional file 1: Table S3 (Total in-hospital costs by hospital) and Additional file 1: Table S4 (Average per-episode total inpatient costs by hospital)). After adjusting for length of stay and patient

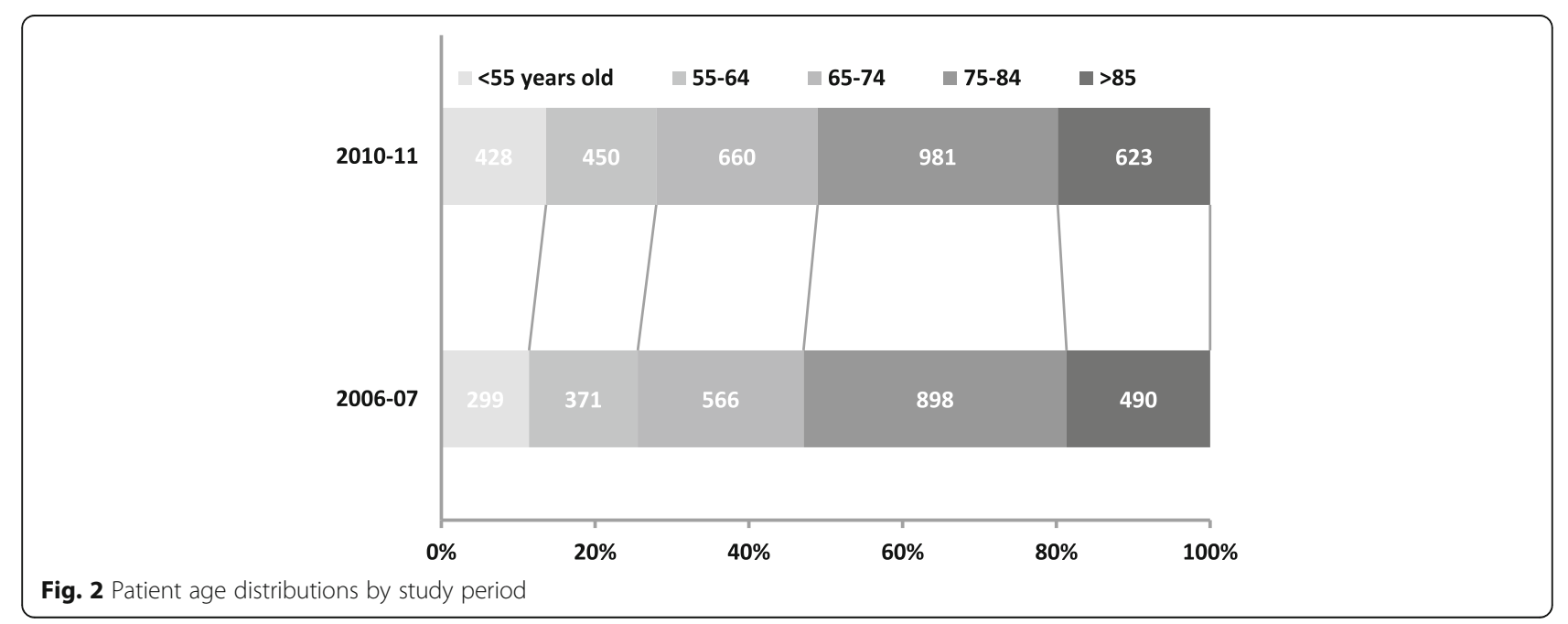


Table 2 Summary results by stroke type and year

\begin{tabular}{|c|c|c|c|c|c|c|c|c|c|c|}
\hline & \multicolumn{2}{|c|}{ Ischaemic stroke } & \multicolumn{2}{|c|}{ Intracerebral haemorrhage } & \multicolumn{2}{|c|}{ Stroke, not specified } & \multicolumn{2}{|l|}{ TIA } & \multicolumn{2}{|l|}{ Total } \\
\hline & 2006-07 & 2010-11 & 2006-07 & 2010-11 & 2006-07 & 2010-11 & 2006-07 & 2010-11 & 2006-07 & 2010-11 \\
\hline Episodes of care & 1110 & 1365 & 273 & 312 & 456 & 393 & 785 & 1072 & 2624 & 3142 \\
\hline$\Delta \%$ & & $23 \%$ & & $14 \%$ & & $-14 \%$ & & $37 \%$ & & $20 \%$ \\
\hline Average LOS & 10.6 & 7.9 & 7.8 & 6.5 & 6.4 & 5.5 & 2.8 & 2.6 & 7.3 & 5.7 \\
\hline$\Delta \%$ & & $-25 \%+$ & & $-17 \%$ & & $-13 \%$ & & $-9 \%$ & & $-22 \%+$ \\
\hline \multicolumn{11}{|l|}{ Costing data ${ }^{a}$} \\
\hline Total costs ('000 s) & $\$ 8109$ & $\$ 9653$ & $\$ 1586$ & $\$ 1960$ & $\$ 2188$ & 1678 & $\$ 1605$ & $\$ 2198$ & $\$ 13,488$ & $\$ 15,589$ \\
\hline$\Delta \%$ & & $19 \%$ & & $24 \%$ & & $-23 \%$ & & $43 \%$ & & $16 \%$ \\
\hline Average per episode cost & $\$ 11,093$ & $\$ 9418$ & $\$ 9275$ & $\$ 8447$ & 7245 & 6454 & $\$ 3171$ & $\$ 3409$ & $\$ 7888$ & $\$ 7115$ \\
\hline$\Delta \%$ & & $-19 \%+$ & & $-20 \%$ & & $-5 \%$ & & $4 \%$ & & $-10 \%+$ \\
\hline
\end{tabular}

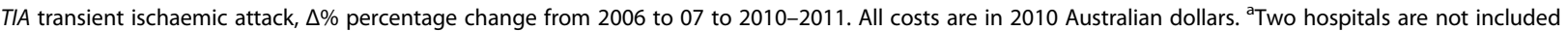
since their clinical costing data were not supplied. $\dagger$ t-test $p<0.01$, all others $p>0.1$

characteristics, there were still differences among the hospitals in 2010-11 between the most expensive (H1: regional site) and least expensive (H3 regional site $p=0.005$, H5 regional site $p<0.001$ ) hospitals in our sample (Fig. 3). Breakdown of mean unadjusted cost per episode in 2010-11 is displayed in Additional file 1: Figure S1. In 2010-11, the main unadjusted cost components overall were nursing (44\%), medical (14\%), allied health (13\%), emergency department (9\%), and imaging (8\%) (Additional file 1: Figure S2). Differences in mean episode costs for each category were compared between 2006-07 and 2010-11. Allied health, emergency, and imaging costs increased $(p<0.01)$ while nursing, medical and pathology costs decreased (all $\mathrm{p}<$ $0.001)$ over the study period. For all other categories, no significant differences were observed.

\section{Discussion}

Clinical facilitators are recognised as important for improving evidence-based practice as part of organisational redesign or achieving task oriented objectives $[10,11,13$, 21]. To our knowledge, we report for the first time on changes in acute hospital costs associated with investment in Facilitators employed to support improvements in the quality of acute stroke care at priority hospitals. Employed for 3-years, the Facilitators in our study had no clinical caseload as part of their role and were able to establish standardised clinical protocols and care plans, and new dedicated stroke units where these were required [13]. The observed changes in practice included increased access to Stroke Units (pre-facilitator 53\%; post $86 \%$ $p<0.001$ ); intravenous thrombolysis (pre-facilitator $2 \%$; post $9 \% \mathrm{p}<0.001)$ and TIA protocols became available in all sites where previously only $50 \%$ provided these [22]. Program success factors included sharing of information between the participating hospitals to reduce duplication of effort and the clinical teams having increased capacity to undertake clinical quality improvement projects.

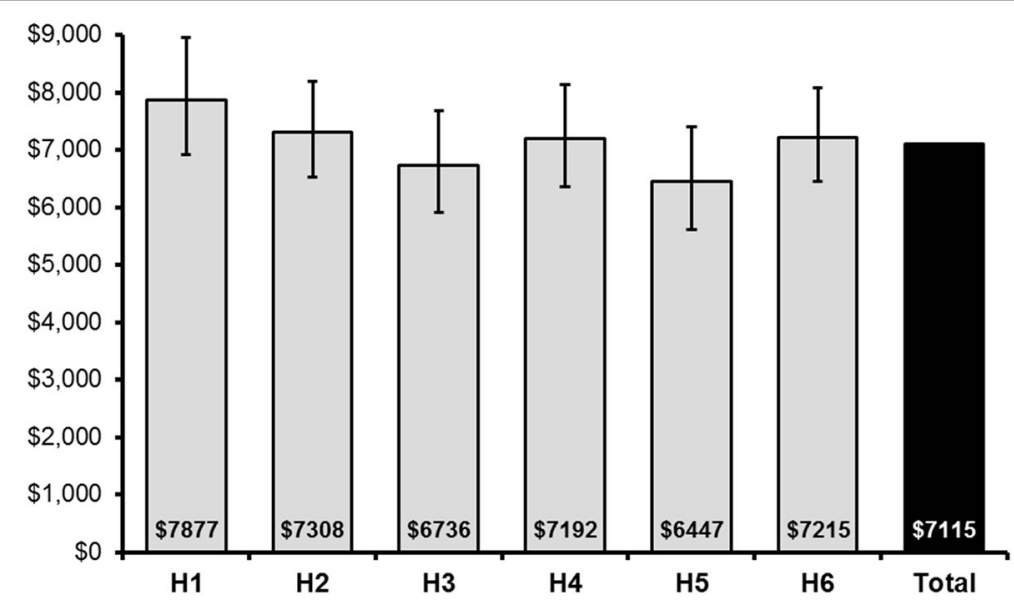

Fig. 3 Average costs (in Australian dollars) per episode for 2010-11 adjusted for patient characteristics and length-of- stay. Estimated marginal means and $95 \%$ confidence intervals of a generalised linear model with gamma distribution and log link adjusting for age, sex, stroke type, and length of stay. $\mathrm{H}=$ hospital. hHospital $\mathrm{H} 4$ and $\mathrm{H} 6$ were located in the Melbourne metropolitan area (other hospitals are in a regional location within Victoria) 
Overall, indications of greater efficiency and capacity to manage more patients with stroke or TIA as inpatients within the context of fewer bed-days being occupied were found. Despite an observed 20\% increase in patient episodes, the number of bed-days utilised was fewer, but the average cost per bed-day was larger (2006-07 AUD709 per day; 2010-11 AUD878 per day). Reassuringly we found evidence of cost containment, with the average total inpatient costs per-episode decreasing by $10 \%$ over 4 years. The cost containment is explained by reductions in average length of stay, a changing case-mix of patients and the need for hospitals to improve their admission and discharge processes to accommodate greater number of patients. These efforts would have been augmented by the achievements of the Facilitators in creating more streamlined management processes including access to rehabilitation services [13].

Our observed reduction in length of stay averaged $4.1 \%$ over the 5 year study period in our priority hospitals with Facilitators, and provides evidence that not all of this reduction is explained by system-wide downward trends. An average annual decrease of $2.1 \%$ in length of stay between 2007 and 08 and 2011-12 among acute care public hospitals in Australia, and with same-day separations excluded, has been reported [23]. Several explanations for our findings are possible, including that there were proportionally more TIA episodes than stroke episodes, and that patients with TIA had shorter lengths of stay than patients with stroke.

The absolute average cost of an episode decreased $(-6 \%)$. When length of stay was additionally adjusted for, the cost per day increased by $\sim 11 \%$ highlighting that reductions in lengths of stay do not necessarily result in overall savings. This is because approximately $40 \%$ of the variable costs are incurred during the first three days of admission [24]. Reductions in length of stay influence the less expensive days in hospital, and with greater efficiency more patients can be treated, but each bed day is more costly [24]. This was explained by a shift in resource utilisation whereby allied health, emergency, and imaging costs were greater contributors to the overall costs in 2010-11 while nursing, medical and pathology costs, usually associated with ongoing care in hospital decreased for this period when compared with 2006-07 (Additional file 1).

The differences in stroke type patterns may be a consequence of improved documentation and coding, as well as better diagnostic work up [13]. This shift in case-mix may also reflect differences in transfer policies within the geographical areas serviced by these hospitals that have now established stroke units.

There have been very few published studies designed to explore the influence of clinical facilitator roles and hospital costs [25, 26], and none to our knowledge in stroke. In the study by Sakallaris et al. (2000), use of a facilitator early in the process to establish same-day transfer protocols to a cardiac telemetry unit after surgery resulted in cost savings and was achieved without compromising the quality of care (assessed by measuring rates of readmission to the ICU or hospital) [26]. Other related studies include use of clinical pathways. In the most recent systematic review by Rotter and colleagues it was concluded that use of clinical pathways was the most likely explanation for the observed reduced lengths of stay and costs [27]. This is consistent with our findings, which also highlight important efficiency gains despite the increased numbers of admissions. Future research is needed to verify our findings or provide cost-effectiveness evidence against other potential models of facilitation in stroke care. This includes a comparison against stroke coordinator roles that are fully embedded within hospitals [7]. In this way, the value of these alternate options for improving stroke care could more effectively guide policy decision-making.

Strengths of the study include use of comprehensive episode-based costing data for whole financial years obtained using reliably applied clinical costing standards [18] and categorised by resource type and stroke type to provide fuller explanatory information. It has been found that the precision of estimates from the Victorian clinical costing data and generalizability to overall Victorian inpatient care is very good, and that by obtaining costs at an ICD10 rather than diagnostic-related group level, we were more likely to have consistency in classification of patients and costs [28].

Potential limitations include that the evaluation of costs presented here relies on the reliability of ICD-10 discharge codes. However, the method for assigning the nominated ICD-10 discharge codes is unlikely to have changed between these periods. There was also evidence of improved diagnostic classification whereby fewer unspecified strokes were reported in the 2010-11 cohort, but this would not have influenced our overall cost results. In addition, we were unable to follow individual patients and the cost per patient could not be derived since the data were de-identified. It is likely that a small number of individuals $(\sim 4 \%)$ [16] will appear several times in the dataset. Re-hospitalisations may artificially increase the number of episodes and decrease the average costs per episode.

It cannot be assumed that all the benefits observed are fully attributable to the Facilitator program in these hospitals. Nevertheless, the magnitude of the changes observed are unlikely to be fully explained by secular improvements in evidence-based practice. For example, the evidence for the establishment of stroke units has existed since 1993 [29], but poor access to 
stroke units is still an issue in Australia [30]. In our primary results paper related to this program we reported changes in processes of care in the post-facilitator period that were much greater than the contemporaneous equivalent data from all other hospitals in Australia [13]. In the current study, we also noted differences in the average costs per episode treated between hospitals. This may in part be explained by teaching and research activities allocated as indirect costs [18]. Therefore, the metropolitan hospitals (two of the six that provided cost data) may have greater costs than the regional hospitals. Alternatively, regional hospitals may be required to pay greater staff costs due to more use of Visiting Medical Officers and patient transport services for diagnostic tests. The small number of sites in this analysis means that comparisons between the metropolitan and regional sites are only indicative of possible differences in resource use and should be interpreted with caution.

Our results might also be influenced by some cost shifting from the ambulatory and/or primary care sector to the inpatient hospital sector, or from acute hospitals to sub-acute care settings such as rehabilitation. More rapid discharge to rehabilitation or to home settings with community supports may provide cost savings for acute hospitals, but may increase costs within these other services. Moreover, there are now a range of rapid assessment outpatient options for managing TIA which may be more efficient than care in hospitals [31]. In an ideal situation, a full cost evaluation should include follow-up of individual patients through the whole stroke system of care [2].

\section{Conclusions}

These data provide important new information about changes in patterns of resource use in a sample of Victorian hospitals where there has been a strong focus on improving acute stroke care made possible through Facilitators. The findings are relevant for stroke care internationally, and may also be applicable to other conditions where variation in practice exists. Importantly, the results highlight that health service improvements can be achieved against a background of increased activity and rising costs. Given the paucity of data in this area further research is needed to verify these findings and to understand the longer term sustainability impacts of implementing this type of program.

\section{Additional file}

Additional file 1: Appendix 1. Description of data: In this additional file, supplemental Methods are outlined to provide the background to clinical costing methods within hospitals for the state of Victoria

(Australia) and additional Results are presented on information at an individual hospital level or aggregated by stroke type in the supplemental Tables and Figures. (DOC $331 \mathrm{~kb}$ )

\section{Abbreviations}

AUD: Australian dollars; Facilitator: Stroke Network Facilitator; H: Hospital; ICD-10: International classification of diseases; ICH: Intracerebral haemorrhage; ID: Identification; IQR: Interquartile range; TIA: Transient ischaemic attack; VSCN: Victorian Stroke Clinical Network

\section{Acknowledgements}

We thank Karen Moss (Florey Institute of Neuroscience and Mental Health) for her administrative assistance with the initial draft of the manuscript. We are grateful to the Stroke Foundation and the hospitals for providing permission to use their National Acute Services Audit data for this evaluation. We acknowledge the assistance of Gregory Dowling from the Victorian Department of Health (now known as the Victorian Department of Health and Human Services, Victorian Government) in sourcing the clinical costing data; as well as Lesley Thornton, Kathryn Whitfield, Janelle Devereux and Adele Mollo.

\section{Funding}

This project was funded by the Victorian Department of Health and Human Services, Victorian Government, through the Victorian Stroke Clinical Network (VSCN) as part of implementation of the Stroke Care Strategy for Victoria. The VSCN commissioned this independent research by The Florey and led by DAC. DAC and AM received research fellowship grants from the National Health and Medical Research Council (DAC: 1063761 co-funded by Heart Foundation; AM: 1091418). The Florey acknowledges support from the Victorian Government, in particular funding from Operational Infrastructure Support Grant.

\section{Availability of data and materials}

The data that support the findings of this study are available from Victorian Department of Health and Human Services but restrictions apply to the availability of these data, which were used under license for the current study, and so are not publicly available. Data are however available from the authors upon reasonable request and with permission of Victorian

Department of Health and Human Services.

\section{Authors' contributions}

DAC contributed to the conception and design of the work, substantial contributions to the data acquisition, analyses, interpretation of data, and drafting of the manuscript. HMD contributed to the design of the work, revision of the manuscript. SD contributed to the conception and design of the work, revision of the manuscript. CFB contributed to the conception and design of the work, revision of the manuscript. AM contributed to the conception and design of the work, substantial contributions to the data acquisition, analyses, interpretation of data, and revision of the manuscript. All authors read and approved the final manuscript.

\section{Ethics approval and consent to participate}

This study was approved by Monash University Human Research Ethics Committee (CF15/3162-2,015,001,349). Individual patient consent was not obtained for this study. The need for consent was waived as we have used anonymised data for a secondary purpose.

\section{Consent for publication}

Not applicable.

\section{Competing interests}

The authors declare that they have no competing interests.

\section{Publisher's Note}

Springer Nature remains neutral with regard to jurisdictional claims in published maps and institutional affiliations.

\section{Author details}

${ }^{1}$ Translational Public Health and Evaluation Division, Stroke and Ageing Research, Department of Medicine, School of Clinical Sciences at Monash Health, Monash University, Clayton, Australia. ${ }^{2}$ Stroke Division, Florey Institute 
of Neuroscience and Mental Health, University of Melbourne, Heidelberg, Australia. ${ }^{3}$ System Design, Planning \& Decision Support Unit, Policy \& Planning Branch, Department of Health and Human Services, Melbourne, Australia. ${ }^{4}$ Eastern Health Clinical School, Monash University, Box Hill, Australia. ${ }^{5}$ Department of Medicine, Royal Melbourne Hospital, University of Melbourne, Parkville, Australia. ${ }^{6}$ Neurocenter, Helsinki University Hospital, Helsinki, Finland.

Received: 23 July 2017 Accepted: 18 December 2018

Published online: 18 January 2019

\section{References}

1. Gloede TD, Halbach SM, Thrift AG, Dewey HM, Pfaff H, Cadilhac DA. Longterm costs of stroke using 10-year longitudinal data from the north East Melbourne stroke incidence study. Stroke. 2014;45(11):3389-94.

2. Meretoja A, Kaste M, Roine RO, Juntunen M, Linna M, Hillbom M, Marttila R, Erila T, Rissanen A, Sivenius J, et al. Direct costs of patients with stroke can be continuously monitored on a national level: performance, effectiveness, and costs of treatment episodes in stroke (PERFECT stroke) database in Finland. Stroke. 2011;42(7):2007-12.

3. Stroke Unit Trialists Collaboration. Organised inpatient (stroke unit) care for stroke. Cochrane Database Syst Rev. 2013;9:CD000197.

4. Cadilhac DA, Ibrahim J, Pearce DC, Ogden KJ, McNeill J, Davis SM, Donnan GA. Multicenter comparison of processes of care between stroke units and conventional care wards in Australia. Stroke. 2004;35(5):1035-40.

5. Moodie M, Cadilhac D, Pearce D, Mihalopoulos C, Carter R, Davis S, Donnan G. Economic evaluation of Australian stroke services: a prospective, multicenter study comparing dedicated stroke units with other care modalities. Stroke. 2006;37(11):2790-5.

6. National Stroke Foundation. National Stroke Audit Clinical Report: aecute services. Melbourne: National Stroke Foundation; 2013. p. 64.

7. Cadilhac DA, Purvis T, Kilkenny MF, Longworth M, Mohr K, Pollack M, Levi CR. On behalf of New South Wales strokes services CoordinatingCommitee, Agency for Clinical Innovation: evaluation of rural stroke services: does implementation of coordinators and pathways improve care in rural hospitals? Stroke. 2013;44(10):2848-53.

8. Kwan J, Sandercock P. In-hospital care pathways for stroke. Cochrane Database Syst Rev. 2004;4:CD002924.

9. Helfrich CD, Damschroder L, Hagedorn HJ, Daggett GS, Sahay A, Ritchie M, Damush T, Guihan M, Ullrich PM, Stetler CB. A critical synthesis of literature on the promoting action on research implementation in health services (PARIHS) framework. Implement Sci. 2010;5:82.

10. Stetler $C B$, Damschroder $L$, Helfrich $C D$, Hagedorn $H J$. A guide for applying a revised version of the PARIHS framework for implementation. Implement Sci. 2011;6:99.

11. Stetler CB, Legro MW, Rycroft-Malone J, Bowman C, Curran G, Guihan M, Hagedorn $\mathrm{H}$, Pineros $\mathrm{S}$, Wallace CM. Role of "external facilitation" in implementation of research findings: a qualitative evaluation of facilitation experiences in the veterans health administration. Implement Sci. 2006;1:23.

12. Purvis T, Moss K, Denisenko S, Bladin C, Cadilhac DA. Implementation of evidence-based stroke care: enablers, barriers, and the role of facilitators. J Multidiscip Healthc. 2014;7:389-400.

13. Purvis T, Moss K, Francis L, Borschmann K, Kilkenny MF, Denisenko S, Bladin CF, Cadilhac DA. Benefits of clinical facilitators on improving stroke care in acute hospitals: a new programme for Australia. Intern Med J. 2017:47(7):775-84

14. Victorian Government Department of Human Services. Stroke care strategy for Victoria. Melbourne: Victorian Government; 2007. p. 158

15. Harris D, Cadilhac D, Hankey GJ, Hillier S, Kilkenny M, Lalor E. National Stroke Audit: the Australian experience. Clin Audit. 2010;2:25-31.

16. Cadilhac DA, Lannin NA, Anderson CS, Andrew N, Kim J, Kilkenny M, Fung F, Grabsch B, Levi C, Faux S, et al. The Australian stroke clinical registry annual report 2013. Heidelberg: The Florey Institute of Neuroscience and Mental Health; 2014. p. 59.

17. Victorian Department of Health. Victorian health services policy and funding guidelines 2010-11 - technical guidelines. Melbourne: Victorian Department of Health; 2010. p. 98.

18. Victorian Department of Health. Victorian cost data collection: business rules for reporting 2010-11 cost data version 3.6. Melbourne: Victorian Department of Health; 2012. p. 26.
19. Australian Institute of Health and Welfare. Health expenditure Australia 2010-11. Canberra: AlHW; 2012. p. 185.

20. Prices and purchasing power parities. [ http://stats.oecd.org/Index. aspx?DatasetCode=MEI_PRICES ].

21. Purvis T, Kilkenny MF, Middleton S, Cadilhac DA. Influence of stroke coordinators on delivery of acute stroke care and hospital outcomes: an observational study. Int J Stroke. 2017;13(6):585-91.

22. Purvis $T$, Moss $K$, Francis $L$, Borschmann $K$, Kilkenny MF, Denisenko $S$, Bladin CF, Cadilhac DA. The benefits of clinical facilitators on improving stroke care in acute hospitals: a new program for Australia. Intern Med J. 2017:47(7):775-84.

23. Australian Institute of Health and Welfare. Australian hospital statistics $2011-$ 12. Health services series. Canberra: AlHW; 2013. p. 362.

24. Taheri PA, Butz DA, Greenfield L. Length of stay has minimal impact on the cost of hospital admission. J Am Coll Surg. 2000;191(2):123-30.

25. Placing facilitator in ED cuts avoidable hospital stays. Clin Resour Manag. 2001;2(2):17-20.

26. Sakallaris BR, Halpin LS, Knapp M, Sheridan MJ. Same-day transfer of patients to the cardiac telemetry unit after surgery: the rapid after bypass Back into telemetry (RABBIT) program. Crit Care Nurse. 2000;20(2):50-5 59-63, 65-58.

27. Rotter T, Kinsman L, James E, Machotta A, Willis J, Snow P, Kugler J. The effects of clinical pathways on professional practice, patient outcomes, length of stay, and hospital costs: Cochrane systematic review and metaanalysis. Eval Health Prof. 2012;35(1):3-27.

28. Jackson T. Cost estimates for hospital inpatient care in Australia: evaluation of alternative sources. Aust N Z J Public Health. 2000;24(3):234-41.

29. Langhorne P, Williams BO, Gilchrist W, Howie K. Do stroke units save lives? Lancet. 1993;342(8868):395-8.

30. National Stroke Foundation. National Stroke Audit: acute services report 2015. Melbourne: National Stroke Foundation; 2015. p. 37.

31. Sanders L, Cadilhac DA, Srikanth V, Pei Chong C, Phan TG. Is nonadmissionbased care for TIA patients cost-effective? A microcosting study. Neurol Clin Pract. 2015;5(1):58-66.

Ready to submit your research? Choose BMC and benefit from:

- fast, convenient online submission

- thorough peer review by experienced researchers in your field

- rapid publication on acceptance

- support for research data, including large and complex data types

- gold Open Access which fosters wider collaboration and increased citations

- maximum visibility for your research: over $100 \mathrm{M}$ website views per year

At BMC, research is always in progress.

Learn more biomedcentral.com/submissions 